\title{
Application of chromosomal microarray analysis in products of miscarriage
}

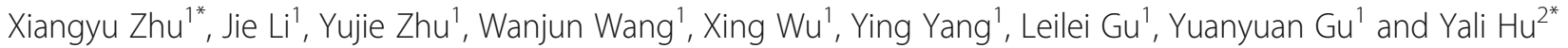

\begin{abstract}
Background: Chromosomal abnormality is one of the major cause of spontaneous abortion. Most available guidelines suggest genetic testing after three miscarriages, which has been proved to be difficult to adhere to and somewhat of low cost-effectiveness. As chromosomal microarray analysis has been recommended to be applied on miscarriage products, we managed a retrospective study on our experience investigate the potential impact of this technique on previous guidelines and our present management on miscarried couples and products.

Results: Of the 405 products of conceptions, the overall detection rate of pathogenetic results was 55.3\% (224/405), including 7.1\% (16/224) copy number changes which could be missed by conventional karyotyping analysis. Of the 222 natural conception samples, abnormal genetic results were found in 126 cases (56.8\%). The detection rate in the assistant reproductive treatment group was 53.6\% (98/183). No significant difference was found between these two groups ( $p=0.645, O R=1.110$ with $95 \% \mathrm{Cl}: 0.713-1.726)$. The detection rate was $53.2 \%$ (75/141) in 141 productof-conceptions (POCs) of mothers with adverse pregnancy histories. Of the 264 POC samples of mothers without abnormal pregnancy histories, 56.4\% (149/264) were genetically abnormal. The detection rate and maternal age between these two groups were all compatible.
\end{abstract}

Conclusions: Chromosomal microarray testing should be referred to couples at their first miscarriage regardless of the way how they get pregnant.

Keywords: Chromosomal microarray analysis, Miscarriage, Spontaneous abortion, Microarray, Genetic testing

\section{Background}

Pregnancy loss at any gestational age could be a catastrophic incident for the woman and her family. Seeking the potential cause is an effective way to estimate the recurrent risk and to take precautions to avoid another pregnancy loss. Though not fully deciphered, a good bunch of etiologies have been established to be related to pregnancy loss, among which, chromosomal abnormalities or genetic imbalances of the embryos or fetus have long been recognized as one of the major cause of miscarriage ( $<20$ weeks' gestation). However, established in cytogenetic ages, most available practical guidelines mainly suggest genetic testing on a third miscarriage $[2,11,23]$. Even the most recent guidelines did not change the opinion [26].

\footnotetext{
*Correspondence: xiangyuzhu82@sina.com; glyyhuyali@163.com

${ }^{1}$ Department of Obstetrics and Gynecology, Nanjing University Medical School Affiliated Nanjing Drum Tower Hospital, Nanjing 210008, Jiangsu, China

${ }^{2}$ Department of Obstetrics and Gynecology, Nanjing Drum Tower Hospital, the Affiliated Hospital of Nanjing University Medical School, Nanjing 210008, Jiangsu, China
}

With traditional cytogenetic techniques, culture failure due to microbial contamination and tissue inactivation was reported as high as $21-25 \%$ when applied to the product-of-conception specimens [28]. Maternal cell contamination and selected cell line proliferation might distort the real condition of fetal karyotype [20]. Resolution and potentially subjective judgment of the chromosomal alternation is another issue of this technique. All these drawbacks might be the handicap of the application and be recommended to a "recurrent" miscarriage.

However, due to the anxiety of the patients suffering from the miscarriage and other reasons, some investigations revealed that adhesion to guidelines was not ideal for both patients and professionals [12, 29, 30]. On the other hand, psychological stress and depression caused by miscarriage might be otherwise a high risk factor of miscarriage $[17,19,31]$. Thus we are considering if there's something we could be done to improve the situation.

Thanks to the dramatic improvement in genetic testing techniques and reduced cost, we are now able to 
detect submicroscopic imbalances on a whole genome scale at a much lower price. Chromosomal microarray analysis (CMA) is one of the most prestigious technique. As a DNA-based technique, CMA is free form the drawbacks of traditional cytogenetic testing. What's more, as CMA could reveal copy number changes over $400 \mathrm{~kb}$ (depending on different platforms that applied) in the scale of whole genome simultaneously, findings of submicroscopic copy number variations $(\mathrm{CNVs})$ were reported as another advantage over traditional techniques. Levy B [16] found 1.6\% pathogenic CNVs in their cohort. Other researchers found an additional $5.7-13 \%$ pathogenic CNVs in karyotypingly normal product-of-conception (POC) specimens [10, 22, 33]. As a result, CMA was generally accepted as a powerful technique to detect genetic imbalances in POC samples.

In this study, by reviewing our microarray data, we are going to confirm the effectiveness of CMA on POC samples, and further investigate the potential impact of this technique on previous guidelines and our present management on miscarried couples and products.

\section{Results}

\section{Subject features and general findings}

Of the 405 products of conceptions, gestational ages ranged from 5 weeks to 19 weeks and 6 days. Three hundred and sixty-seven (367/405,90.6\%) POC samples were before 12 weeks and 6 days, or early miscarriage. Thirty-eight $(38 / 405,9.4 \%)$ POC samples were obtained from demised fetus between 13 gestational weeks and 19 gestational weeks and 6 days, also called late miscarriage. Maternal ages of these products-of-conception samples ranged from 20 to 46 $(31.33 \pm 4.74)$ years old

The overall detection rate of pathogenetic findings was 55.3\% (224/405). Maternal ages of the genetically abnormal POC samples were significantly older than the normal group $(p<0.001$, see Table 1$)$. Single aneuploidies were the most common abnormality, accounting for $77.2 \%(173 / 224)$ of all the abnormalities, among which, the most affected chromosomal aneuploidies were trisomy-16, trisomy-22 and monosomy X, accounting for $14.7 \%$ (33/224, including two cases of trisomy-16 with microscopic CNVs), $12.9 \%(29 / 224)$ and $11.6 \%(26 / 224)$ respectively (Table 2$)$. CNV was the fourth most common abnormal finding, accounting for $7.1 \%(16 / 224)$ of the total abnormality (Table 2).
Notably, CNV mentioned in this paper is not necessarily submicroscopic. Partial trisomy or monosomy that might be difficult to figure out under microscope was also defined as $\mathrm{CNV}$ (Table 4).

\section{Genetic findings in subgroups}

According to the way how the women got pregnant at "this" time, we divided the samples into two groups: products of natural conception (NC) and products of assisted reproductive technique (ART). Of the $222 \mathrm{NC}$ samples, abnormal genetic results were found in 126 cases (56.8\%), the mean maternal age in this group was $30.51 \pm 4.47$ years old. The detection rate in the ART group was 53.6\% (98/183) (Table 3). Mean maternal age of the ART group was $32.30 \pm$ 4.68, significantly older than the NC group mother. When adjusted by the maternal age, the detection rate showed no significant difference between these two groups $(p=0.645, \quad O R=1.110$ with $95 \% \mathrm{CI}$ : 0.713-1.726). Among the ART group, 17 cases were products of intracytoplasmic sperm injection (ICSI) and 166 cases were products of in vitro fertilization (IVF). The detection rates were comparative between ICSI and IVF group (10/17 Vs 88/68, $p>0.5)$.

According to pregnancy histories, we categorized the samples into two groups, with adverse pregnancy history (APHs) and without APHs. According to times of APHs, we further divided this group into two groups. One group included the POC samples of women who experienced more than two previous pregnancies with adverse outcome (indicated as subgroup A), the other included POC samples of women with only one adverse pregnancy (indicated as subgroup B). As a result, 141 cases were POCs of mothers with APHs. The detection rate was 53.2\% (75/141) in this whole group, with $52.1 \%(38 / 73)$ and $54.4 \%$ (37/68) detection rate for subgroup A and B respectively (Table 3). Of the 264 POC samples of mothers without APHs, 56.4\% (149/264) were genetically abnormal. The detection rate and maternal age between these two groups were all compatible (Table 1 and Table 2). No difference exists between either two groups (Table 3).

Two hundred six abnormal results were found in miscarriages before 12 weeks and 6 days, accounting for $56.1 \%$ cases $(206 / 367)$ of this early pregnancy loss

Table 1 Maternal age in different groups

\begin{tabular}{|c|c|c|c|c|c|c|}
\hline & IVF & $\mathrm{NC}$ & Without APHs & With APHs & Normal & Abnormal \\
\hline Maternal age & $32.30 \pm 4.68$ & $30.51 \pm 4.47$ & $31.56 \pm 4.93$ & $30.91 \pm 4.40$ & $30.42 \pm 4.30$ & $32.00 \pm 4.98$ \\
\hline$p$-value & $<0.001$ & & 0.19 & & $<0.001$ & \\
\hline
\end{tabular}

IVF in vitro fertilization, $N C$ natural conception, $A P H s$ adverse pregnancy histories 
Table 2 Detailed spectrum of genomic imbalances in products of conception

\begin{tabular}{|c|c|c|c|c|c|c|c|c|c|c|}
\hline \multirow[t]{2}{*}{ Genomic imbalances } & & & \multirow[t]{2}{*}{ ART } & \multirow[t]{2}{*}{ NC } & \multirow[t]{2}{*}{ Without APHs } & \multicolumn{2}{|c|}{ With APHs } & \multirow[t]{2}{*}{$13-19+6$ GWs } & \multirow[t]{2}{*}{$<13 \mathrm{GWS}$} & \multirow[t]{2}{*}{ Total } \\
\hline & & & & & & $\mathrm{A}$ & B & & & \\
\hline \multirow[t]{22}{*}{ Single aneuploidy } & Autosomal trisomy & 2 & 2 & 3 & 5 & 0 & 0 & 0 & 5 & 5 \\
\hline & & 3 & 0 & 2 & 2 & 0 & 0 & 1 & 1 & 2 \\
\hline & & 4 & 1 & 3 & 1 & 1 & 2 & 0 & 4 & 4 \\
\hline & & 5 & 1 & 0 & 1 & 0 & 0 & 0 & 1 & 1 \\
\hline & & 6 & 0 & 1 & 0 & 1 & 0 & 0 & 1 & 1 \\
\hline & & 7 & 3 & 3 & 5 & 1 & 0 & 0 & 6 & 6 \\
\hline & & 8 & 2 & 3 & 4 & 1 & 0 & 0 & 5 & 5 \\
\hline & & 9 & 1 & 1 & 2 & 0 & 0 & 0 & 2 & 2 \\
\hline & & 10 & 1 & 1 & 2 & 0 & 0 & 0 & 2 & 2 \\
\hline & & 11 & 1 & 1 & 1 & 0 & 1 & 0 & 2 & 2 \\
\hline & & 12 & 1 & 1 & 1 & 1 & 0 & 0 & 2 & 2 \\
\hline & & 13 & 1 & 9 & 3 & 5 & 2 & 1 & 9 & 10 \\
\hline & & 14 & 0 & 1 & 0 & 1 & 0 & 0 & 1 & 1 \\
\hline & & 15 & 6 & 5 & 6 & 3 & 2 & 0 & 11 & 11 \\
\hline & & $16^{a}$ & 15 & 18 & 19 & 4 & $10^{\mathrm{a}}$ & 1 & 32 & 33 \\
\hline & & 18 & 3 & 4 & 4 & 1 & 2 & 3 & 4 & 7 \\
\hline & & 20 & 3 & 4 & 5 & 1 & 1 & 0 & 7 & 7 \\
\hline & & 21 & 6 & 6 & 11 & 1 & 0 & 2 & 10 & 12 \\
\hline & & 22 & 13 & 16 & 23 & 3 & 3 & 1 & 28 & 29 \\
\hline & Other aneuploidy & monosomy 21 & 3 & 1 & 4 & 0 & 0 & 0 & 4 & 4 \\
\hline & & monosomy $X$ & 13 & 13 & 17 & 3 & 6 & 5 & 21 & 26 \\
\hline & & $X X Y$ & 0 & 1 & 0 & 0 & 1 & 0 & 1 & 1 \\
\hline CNV & & CNV & 7 & 9 & 11 & 2 & 3 & 1 & 15 & 16 \\
\hline \multirow[t]{2}{*}{ Multiple aneuploidy } & & Double trisomy & 7 & 3 & 7 & 2 & 1 & 0 & 10 & 10 \\
\hline & & Trisomy 22 with monosomy $X$ & 2 & 0 & 2 & 0 & 0 & 0 & 2 & 2 \\
\hline \multirow[t]{5}{*}{ Triploidy } & triploidy & triploidy & 3 & 10 & 9 & 3 & 1 & 3 & 10 & 13 \\
\hline & Hyper-triplody & Triploidy with tetrasomy $X$ & 0 & 1 & 1 & 0 & 0 & 0 & 1 & 1 \\
\hline & & Triploidy with tetrasomy 16 & 0 & 1 & 1 & 0 & 0 & 0 & 1 & 1 \\
\hline & & Triploidy with tetrasomy 8 and 14 & 0 & 1 & 0 & 0 & 1 & 0 & 1 & 1 \\
\hline & Hypo-triplody & Triploidy with disomy 13 & 0 & 2 & 0 & 1 & 1 & 0 & 2 & 2 \\
\hline UPD & & UPD & 0 & 2 & 0 & 2 & 0 & 0 & 2 & 2 \\
\hline \multirow[t]{3}{*}{ Mosaicism } & & Mosaic trisomy 6 & 1 & 0 & 1 & 0 & 0 & 0 & 1 & 1 \\
\hline & & Trisomy 16 with mosaic trisomy 13 & 1 & 0 & 1 & 0 & 0 & 0 & 1 & 1 \\
\hline & & Mosaic trisomy 18 & 1 & 0 & 0 & 1 & 0 & 0 & 1 & 1 \\
\hline Total abnormal & & & 98 & 126 & 149 & 38 & 37 & 18 & 206 & 224 \\
\hline
\end{tabular}

A: subgroup A, POC samples of women who experienced more than two previous pregnancies with adverse outcome; B: subgroup B, POC samples of women who experienced one adverse pregnancy history

$A R T$ assisted reproductive treatment, NC natural conception, $A P H x$ adverse pregnancy history, GW gestational week, CNV copy number variance, UPD uniparental disomy ${ }^{a}$ two cases were trisomy-16 with CNVs

group. $47.3 \%(18 / 38)$ POC samples of 13 to 19 weeks and 6 days of gestational age were abnormal. Pathogenic CNVs were found in 4.1\% (15/367) early miscarriages and $2.6 \%(1 / 38)$ in late miscarriages (Table 2). None of these detection rates showed significant differences between comparable groups.

\section{Discussion}

Additional yields support the use of CMA for POC samples

On chromosomal level, CMA results are highly accordant to cytogenetic results, and with a higher report rate [16]. What's more, an additional $5.7-13 \%$ pathogenic 
Table 3 Genetic abnormal findings in different groups

\begin{tabular}{lcccllrr}
\hline & ART & NC & Without APHx & $\geq 3$ APHx & With 2 APHx & $<13 G W s$ & $13-19+6$ GWs \\
\hline Abnormal & 98 & 126 & 149 & 38 & 37 & 206 & 18 \\
Normal & 85 & 96 & 115 & 35 & 31 & 161 & 20 \\
$p$-value $(95 \% \mathrm{Cl})$ & $0.578(0.604-1.326)$ & 0.294 & & & $0.301(0.728-2.776)$ \\
\hline
\end{tabular}

CNVs in karyotypingly normal POC specimens [10, 22, 33]. Based on a more than 2000 cohort study, Dr. Levy and his colleagues provided a level III evidence to support the use of CMA for the cytogenetic evaluation of miscarriage specimens [16]. According to our study, we detected 5 cases with CNVs $(1.2 \%)$ that less than $10 \mathrm{Mb}$ (Table 4: case 12-16). This detection rate of submicroscopic CNVs is compatible to previous reports [10, 22, 33].

Moreover, CMA may be more sensitive and accurate to detect imbalanced chromosomal rearrangement. POC, as a sort of proband, could be used as a perfect clue to uncover the potential existence of a balanced translocation in either of the parent. According to our CMA results, we deduced that 5 miscarriages may be consequences of recombination of balanced translocations or inversions in either of the parents (Table 4: case 2, 3, 5, 6 and 9). Two cases might be caused by complex chromosome rearrangement (Table 4: case 1 and 8). Other 4 miscarriages might be due to de novo mutation or mosaic conditions of their parents (Table 4: case 4, 7 , 10 and 11). As these 11 cases might not be effectively recognized under microscope for the concealed nature of these chromosomal change, we grouped them as CNVs together with the previous 5 truly $\mathrm{CNV}$ cases to highlight the potential advantages of CMA.

In our present data, 2 UPD cases were revealed. The ability to detect uniparental disomy (UPD) with single nucleotide polymorphism (SNP) markers is another advantage of the microarray method we have used. As risk of rare imprinting disorders raises after ART [27], microarray could be applied as a useful tool to make further research.

\section{Pregnancy loss and adverse pregnancy history}

Many researchers suggested that a history of trisomy conception was a risk factor of future pregnancies being trisomic, especially for women younger than 35 years old [1, 5, 9, 32]. A higher risk of nondisjunction was thought to be existed in these women [32]. However, according to our study, no difference of genetic abnormal rate was found between either two groups of patients without APHs, one adverse pregnancy and more than one adverse pregnancy.

Miscarriage is a multifactorial situation. In a large cohort study, Bhattacharya suggested that the risk of a

Table 4 CNVs found in pregnancy loss

\begin{tabular}{|c|c|c|c|c|c|c|}
\hline No. & MA & GW & ART & APHx & CMA result & Size $(\mathrm{Mb})$ \\
\hline 1 & 43 & 6 & Y & $\mathrm{N}$ & arr [hg19] 8p23.3p11.1 (158,048-43,824,035) × 1, 8p11.1q24.3 $(43,837,098-146,295,771) \times 3$ & $43.7,102.5$ \\
\hline 2 & 29 & 7 & $\mathrm{~N}$ & N & arr [hg19] 7p22.3p11.2 (162,702-57,780,598) ×3, 17p13.3p11.2 $(525-21,518,996) \times 1$ & $57.6,20.99$ \\
\hline 3 & 36 & 6 & Y & 2 & arr [hg19] 8p23.3p12 $(158,048-30,234,334) \times 1,8 q 22.1 q 24.3(97,466,303-146,295,771) \times 2-3$ & $30,48.8$ \\
\hline 4 & 28 & 8 & $\mathrm{~N}$ & 3 & arr [hg19] 19q11q13.43 $(28,271,417-58,956,816) \times 3$ & 30.7 \\
\hline 5 & 27 & 11 & Y & N & arr [hg19] 8p23.3p12 $(158,048-29,402,007) \times 1,8 p 12(30,393,410-34,277,594) \times 3$ & $30,3.88$ \\
\hline 6 & 27 & 8 & Y & N & $\begin{array}{l}\text { arr [hg19] 18p11.21q11.2(12,520,909-19,043,748) ×3, 18q21.2q23 }(52,653,009-78,013,728) \\
\times 1,19 q 13.41 \mathrm{q} 13.43(54,872,973-58,956,816) \times 3\end{array}$ & $6.5,25.4,4.1$ \\
\hline 7 & 33 & 6 & Y & N & arr [hg19] 15q25.3q26.3 $(87,014,450-102,429,040) \times 1$ & 25.4 \\
\hline 8 & 22 & 7 & $\mathrm{~N}$ & $\mathrm{~N}$ & arr [hg19] 11q22.1q24.2 (102,024,970-127,356,904) ×3, 11q24.3q25 $(128,670,114-134,937,416) \times 1$ & $25.33,6.267$ \\
\hline 9 & 33 & 7 & $\mathrm{~N}$ & 3 & arr [hg19] 1p36.33p36.11 $(849,466-24,454,688) \times 3,7 q 36.2 q 36.3(153,468,186-159,119,707) \times 1$ & $23.6,5.65$ \\
\hline 10 & 29 & 7 & Y & 2 & arr [hg19] 10q21.2q22.2 $(61,408,306-76,588,258) \times 1$ & 15.18 \\
\hline 11 & 25 & 5 & $\mathrm{~N}$ & $\mathrm{~N}$ & arr [hg19] 18p11.32p11.21 $(136,227-15,099,116) \times 1$ & 14.96 \\
\hline 12 & 30 & 6 & $\mathrm{~N}$ & $\mathrm{~N}$ & arr [hg19] 1p36.33p36.22 $(849,466-10,391,536) \times 1$ & 9.5 \\
\hline 13 & 30 & 11 & $\mathrm{~N}$ & $\mathrm{~N}$ & arr [hg19] 10q22.3q23.2 $(81,630,468-88,785,190) \times 3$ & 7.16 \\
\hline 14 & 21 & 10 & N & 2 & arr [hg19] 22q11.21 $(18,648,855-21,800,471) \times 1$ & 3.1 \\
\hline 15 & 25 & 12 & Y & $\mathrm{N}$ & arr [hg19] 22q11.21 $(18,916,842-21,163,516) \times 3$ & 3.1 \\
\hline 16 & 29 & 9 & Y & $\mathrm{N}$ & arr [hg19] 22q11.22q11.23 $(22,331,458-23,652,518) \times 3$ & 1.32 \\
\hline
\end{tabular}


further miscarriage increased in women who had one and two miscarriages regardless of the etiology [4]. Researchers have also found that women with recurrent pregnancy loss (RPL) suffered from more psychological stress and depression $[7,15,19]$, which could trigger a negative feedback [8] threatening subsequent conception $[17,19,31]$.

Bernardi and her colleagues' research [3] revealed that selective RPL evaluation based upon chromosome testing was a cost-saving strategy when compared to universal RPL evaluation. As a further supportive evidence of this opinion, our CMA result have found 2 cases (Table 3: case 4 and 5) of genetic imbalance which was quite possibly be the consequence of recombination of a balanced translocation existed in either parent. The detection of this translocation could be finely defined as the etiology of potential recurrent miscarriage [24, 25].

Considering the benefits of CMA, the impact of spontaneous miscarriage on subsequent pregnancy and the potential effect-economic benefits, we suggest that CMA test on POC-sample should be recommended to couples who suffered from their first miscarriage.

\section{Assisted reproductive technique versus natural conception}

ART is a major therapy method in many infertile couples. Only a few studies with limited sample volume were concerned on the risk of chromosomal abnormalities after ART treatment. And to some pitfalls, the results were still controversial. Campos-Galindo et al. [6] tested 189 samples from ART or NC pregnancies with KaryoLite ${ }^{\mathrm{Tm}} \mathrm{BoBs}^{\mathrm{rm}}$, and they observed a considerably higher rate of aneuploidy in the ART group using the patient's own oocytes. However, the maternal age of this group was significantly higher. Some detailed studies found no increased risk of chromosomal abnormalities due to ART [14, 18]. A recent meta-analysis had come to the same conclusion that no statistical difference was existed in risk of chromosomally abnormal miscarriage between ART and NC groups [21]. Our result as well indicated no increased burden of genetic abnormalities occurred after ART (data were adjusted by maternal age). These studies might relieve us a bit of the worries about ART. However, as limited by the sample size, we didn't further divide them according to specific technique of ART they applied. More samples are need to make a conclusive result.

\section{Conclusion}

According to our experience and of previous researches, CMA presented as reliable and comprehensive technique to detect genetic imbalances in POC samples and stillbirths. As no significant difference was found in ART group versus $\mathrm{NC}$ group and pregnancy with APHs versus without APHs, genetic testing should be reoffered to couples at their first miscarriage, and couples undergo ART could be a little relieved of the additional risk of chromosomal abnormalities.

\section{Methods \\ Materials}

From January 2014 to November 2017, reportable results were obtained from 405 POCs, including villi (380 cases) and tissues of fetus while available ( 25 cases). All the samples were collected routinely when parents wanted to seek a genetic etiology of the miscarriages. Consent forms were signed by couples before the tests. Parental peripheral blood specimens were obtained together with the products of conceptions. History of previous pregnancy (s) and the way how they got conceived (natural conception or by assisted reproductive techniques) at this time were recorded. Adverse pregnancy history (APHs) was defined when either condition existed as below: spontaneous miscarriage or stillbirth; termination of pregnancy because of fetal malformation; infantile death because of severe structural abnormalities.

\section{Methods}

All the villi were carefully separated by needles under the anatomical microscope. Any macroscopic blood was washed away from tissues by saline solution. By using four highly polymorphic short tandem repeats, D2S1338, D7S820, D13S317 and D21S11 for specific, maternal cell contamination was ruled out for all the 405 samples.

Chromosomal microarray analysis was performed using the Affymetrix CytoScan platform, which contains both SNP markers and copy markers. All genomic DNA samples were digested, amplified, fragmented, labeled and hybridized to CytoScan $750 \mathrm{~K}$ chips according to the manufacturer's protocol. Raw data was analyzed by ChAS 3.1 software (Affymetrix, USA). Interpretation of the CNVs defined according to the ACMG guidelines [13]. CNVs of unknown significance were further tested on the parents' DNAs by quantitative fluorescent PCR. If it was constitutive in phenotypically normal parent, the $\mathrm{CNV}$ of unknown significance was never thought to be causative and then classified as "normal" in this study.

\section{Statistical analyses}

Statistical analysis was performed using IBM SPSS Statistics, version 21. T-test, regression analysis and Chi-square test were applied in necessary situations. $p<0.05$ was considered statistically significant.

\footnotetext{
Abbreviations

APHs: Adverse pregnancy history; ART: Assisted reproductive technique; CMA: Chromosomal microarray analysis; CNV: Copy number variations; GW: Gestational age; ICSI: Intracytoplasmic sperm injection; IVF: In vitro fertilization; MA: Maternal age; NC: Natural conception; POC: Product-ofconceptions; RPL: Recurrent pregnancy loss; SNP: Single nucleotide polymorphism; UPD: Uniparental disomy
} 


\section{Acknowledgements}

We would like to thank our patients for agreeing to donate their personal data to our study and have these been published.

\section{Funding}

This study is supported by Jiangsu Provincial Medical Youth Talent (QNRC2016030), Grant for Science and Technology Development (201605018) and National Natural Science Foundation of China (CN) (81300490).

\section{Availability of data and materials}

Please contact author for data request.

\section{Authors' contributions}

The corresponding authors are responsible for the study design and analysis and manuscript drafting. YG who was granted the National Science Fundation is another supporter of this research. YZ, WW and LG carried out the microarray experiment. JL, XW and YY were responsible for the genetic counseling and data analysis. All authors read and approved the final manuscript.

\section{Ethics approval and consent to participate}

This study was approved by the Ethics Committee of the Nanjing Drum Tower Hospital.

\section{Consent for publication}

Not applicable.

\section{Competing interests}

The authors declare that they have no competing interests.

\section{Publisher's Note}

Springer Nature remains neutral with regard to jurisdictional claims in published maps and institutional affiliations.

\section{Received: 27 June 2018 Accepted: 8 August 2018} Published online: 17 August 2018

\section{References}

1. Al-Asmar N, Peinado V, Vera M, Remohí J, Pellicer A, Simón C, et al. Chromosomal abnormalities in embryos from couples with a previous aneuploid miscarriage. Fertil Steril. 2012;98:145-50.

2. American College of Obstetricians and Gynecologists (ACOG). Management of recurrent early pregnancy loss. ACOG practice bulletin. Int J Gynaecol Obstet. 2002;78:179-90.

3. Bernardi LA, Plunkett BA, Stephenson MD. Is chromosome testing of the second miscarriage cost saving? A decision analysis of selective versus universal recurrent pregnancy loss evaluation. Fertil Steril. 2012;98:156-61.

4. Bhattacharya S, Townend J, Bhattacharya S. Recurrent miscarriage: are three miscarriages one too many? Analysis of a Scottish population-based database of 151,021 pregnancies. Eur J Obstet Gynecol Reprod Biol. 2010;150:24-7.

5. Bianco K, Caughey AB, Shaffer BL, Davis R, Norton ME. History of miscarriage and increased incidence of fetal aneuploidy in subsequent pregnancy. Obstet Gynecol. 2006;107:1098-102.

6. Campos-Galindo I, Garcia-Herrero S, Martinez-Conejero JA, Ferro J, Simón C, Rubio C. Molecular analysis of products of conception obtained by hysteroembryoscopy from infertile couples. J Assist Reprod Genet. 2015; 32(5):839-48.

7. Cheung $\mathrm{CS}$, Chan $\mathrm{CH}, \mathrm{Ng}$ EH. Stress and anxiety-depression levels following first-trimester miscarriage: a comparison between women who conceived naturally and women who conceived with assisted reproduction. BJOG. 2013;120:1090-7.

8. Craig M. Stress and recurrent miscarriage. Stress. 2001:4:205-13.

9. De Souza E, Halliday J, Chan A, Bower C, Morris JK. Recurrence risks for trisomies 13, 18, and 21. Am J Med Genet A. 2009;149A:2716-22.

10. Dhillon RK, Hillman SC, Morris RK, McMullan D, Williams D, Coomarasamy A, et al. Additional information from chromosomal microarray analysis (CMA) over conventional karyotyping when diagnosing chromosomal abnormalities in miscarriage: a systematic review and meta-analysis. BJOG. 2014;121:11-21.

11. European Society for Human Reproduction and Embryology (ESHRE), Jauniaux E, Farquharson RG, Christiansen OB, Exalto N. Evidence-based guidelines for the investigation and medical treatment of recurrent miscarriage. Hum Reprod. 2006;21:2216-22.
12. Franssen MT, Korevaar JC, van der Veen F, Boer K, Leschot NJ, Goddijn M. Management of recurrent miscarriage: evaluating the impact of a guideline. Hum Reprod. 2007;22:1298-303.

13. Kearney HM, Thorland EC, Brown KK, Quintero-Rivera F, South ST, A Working Group of the American College of Medical Genetics (ACMG) Laboratory Quality Assurance Committee. American College of Medical Genetics standards and guidelines for interpretation and reporting of postnatal constitutional copy number variants. Genet Med. 2011;13:680-5.

14. Kim JW, Lee WS, Yoon TK, Seok HH, Cho JH, Kim YS, et al. Chromosomal abnormalities in spontaneous abortion after assisted reproductive treatment. BMC Med Genet. 2010;11:153.

15. Kolte AM, Olsen LR, Mikkelsen EM, Christiansen OB, Nielsen HS. Depression and emotional stress is highly prevalent among women with recurrent pregnancy loss. Hum Reprod. 2015;30:777-82.

16. Levy B, Sigurionsson S, Pettersen B, Maisenbacher MK, Hall MP, Demko Z, et al. Genomic imbalance in products of conception: single-nucleotide polymorphism chromosomal microarray analysis. Obstet Gynecol. 2014;123:202-9.

17. Li W, Newell-Price J, Jones GL, Ledger WL, Li TC. Relationship between psychological stress and recurrent miscarriage. Reprod BioMed Online. 2012; 25:180-9.

18. Martínez MC, Méndez C, Ferro J, Nicolás M, Serra V, Landeras J. Cytogenetic analysis of early nonviable pregnancies after assisted reproduction treatment. Fertil Steril. 2010;93:289-92.

19. McCarthy FP, Moss-Morris R, Khashan AS, North RA, Baker PN, Dekker G, et al. Previous pregnancy loss has an adverse impact on distress and behaviour in subsequent pregnancy. BJOG. 2015;122:1757-64.

20. Menten B, Swerts K, Delle Chiaie B, Janssens S, Buysse K, Philippe J, et al. Array comparative genomic hybridization and flow cytometry analysis of spontaneous abortions and mors in utero samples. BMC Med Genet. 2009;10:89.

21. Qin JZ, Pang LH, Li MQ, Xu J, Zhou X. Risk of chromosomal abnormalities in early spontaneous abortion after assisted reproductive technology: a metaanalysis. PLoS One. 2013;8:e75953.

22. Rosenfeld JA, Tucker ME, Escobar LF, Neill NJ, Torchia BS, McDaniel LD, et al. Diagnostic utility of microarray testing in pregnancy loss. Ultrasound Obstet Gynecol. 2015:46:478-86.

23. Royal College of Obstetricians and Gynaecologists (RCOG). The investigation and treatment of couples with recurrent miscarriage. 2011. Available online at: http://www.rcog.org.uk.

24. Sugiura-Ogasawara M, Ozaki Y, Sato T, Suzumori N, Suzumori K. Poor prognosis of recurrent aborters with either maternal or paternal reciprocal translocation. Fertil Steril. 2004;81:367-73.

25. Sugiura-Ogasawara M, Ozaki Y, Suzumori N. Management of recurrent miscarriage. J Obstet Gynaecol Res. 2014;40:1174-9.

26. Toth B, Würfel W, Bohlmann MK, Gillessen-Kaesbach G, Nawroth F Rogenhofer $\mathrm{N}$, et al. Recurrent miscarriage: diagnostic and therapeutic procedures. Guideline of the DGGG (S1-level, AWMF registry no. 015/050, December 2013). Geburtshilfe Frauenheilkd. 2015;75:1117-29.

27. Uk A, Collardeau-Frachon S, Scanvion Q, Michon L. Assisted reproductive technologies and imprinting disorders: results of a study from a French congenital malformations registry. Eur J Med Genet. 2018. https://doi.org/10. 1016/j.jmg.2018.05.017

28. van den Berg MM, van Maarle MC, van Wely M, Goddijin M. Genetics of early micscarriage. Biochim Biophys Acta. 2012;1822:1951-9.

29. van den Boogaard E, Hermens RP, Franssen AM, Doornbos JP, Kremer JA, van der Veen F, et al. Recurrent miscarriage: do professionals adhere to their guidelines. Hum Reprod. 2013;28:2898-904.

30. Van Den Boogaard E, Hermens RP, Leschot NJ, Baron R, Vollebergh JH Bernardus RE, et al. Identification of barriers for good adherence to a guideline on recurrent miscarriage. Acta Obstet Gynecol Scand. 2011:90:186-91.

31. Wainstock T, Lerner-Geva L, Glasser S, Shoham-Vardi I, Anteby EY. Prenatal stress and risk of spontaneous abortion. Psychosom Med. 2013a:75:228-35.

32. Warburton D, Dallaire L, Thangavelu M, Ross L, Levin B, Kline J. Trisomy recurrence: a reconsideration based on north American data. Am J Hum Genet. 2004;75:376-85.

33. Zhou Q, Wu SY, Amato K, DiAdamo A, Li P. Spectrum of cytogenomic abnormalities revealed by array comparative genomic hybridization on products of conception culture failure and normal karyotype samples. J Genet Genomics. 2016:43:121-31. 